\title{
Gravitating magnetic monopole in Vaidya geometry
}

\author{
Sushant G. Ghosh $*$ \\ Center for Theoretical Physics, Jamia Millia Islamia, New Delhi - 110025, INDIA \\ L. P. Singh甲 \\ Physics Department, Utkal University, Bhubaneswar 751004 INDIA
}

\begin{abstract}
A magnetic-monopole solution of a non-Abelian gauge theory as proposed by 't Hooft and Polyakov is studied in the Vaidya spacetime. We find that the solutions of Einstein equations generates a geometry of the Bonnor-Vaidya corresponding to magnetically charged null fluid with Higgs field contributing a cosmological term. In the absence of the scalar fields the corresponding $\mathrm{Wu}$-Yang solution of the gauge theory still generates the Bonnor-Vaidya geometry, but with no cosmological term.
\end{abstract}

PACS numbers: 04.20.Jb, 04.70.Bw, 14.80.Hv, 11.15.-q

\section{INTRODUCTION}

One of the most important works on Abelian gauge theories was due to Dirac, who proposed a solution that corresponds to a pointlike magnetic monopole with a singularity string running from the particles position to infinity [1]. This has led to considerable interest among physicists on the possible existence of the magnetic monopole which was further intensified after 't Hooft [2] has proposed solutions for a magnetic monopole which arises as a static solution of the classical equations for Yang-Mills (YM) field coupled to Higgs fields (see also Polyakov [3]). He pointed out that a unified gauge theory in which electromagnetism is embedded in a semisimple gauge group would predict the existence of the magnetic monopole as a soliton with spontaneous symmetry breaking. The 't Hooft-Polyakov magnetic solutions are similar to the $\mathrm{Wu}$ and Yang [4] classical monopole solution to the $\mathrm{SO}(3)$ isospin gauge theory describing a magnetic monopole which is pointlike and has a potential which behaves like $1 / r$ everywhere. It behaves like an Abelian theory at large distance with the gauge-field resembling that due to an Abelian Dirac magnetic monopole of magnetic charge $1 / e$; $e$ is $\mathrm{YM}$ coupling constant. The question that arises naturally is what happens when such monopoles are coupled to gravity. The generalization of the 't Hooft-Polyakov solutions to curved space-time also attracted lot of interest [5-10]. Bais and Russell [ $[6]$ and independently by Cho and Freund [7] found a solution to complete Einstein-Yang-Mills-Higgs (EYMH) system yielding a geometry of ReissnerNordström-de Sitter with the Higgs field contributing to a cosmological term. Yasskin [5] gave a explicit algorithm so that from each solution of the Einstein-Maxwell equations one can set of solutions of EYM equations. A curved-space generalization of the Wu-Yang solution is shown to be a special case of Yasskin's [5] solutions. More recently, using Yasskin's [5] procedure, Mazharimousavi and Halil- soy [11 13 have found a sequence of static spherically symmetric HD-EYM black hole solutions. The remarkable feature of this Wu-Yang ansatz is that the field has no contribution from gradient and instead has pure YM non-Abelian component.

It would be interesting to further consider nonstatic generalization of 't Hooft-Polyakov solutions. It is the purpose of this paper is to obtain an exact nonstatic solution of the Einstein field equations for a 't Hooft - Polyakov solutions in the presence of the null fluid, i.e., a nonstatic curved space-time generalization 't HooftPolyakov solutions in Vaidya geometry. The Vaidya geometry permitting the incorporations of the effects of null fluid offers a more realistic background than static geometries, where all back reaction is ignored. The Vaidya [14] and Bonnor-Vaidya 15] (charged Vaidya) solutions are widely used to model black hole evaporation and to solve the black hole evaporation problem. It is also commonly used as a testing ground for various gravitational scenario and formulation of the cosmic censorship. For a general method of obtaining spherically symmetric solutions in Vaidya geometry, see Ref. [16 18]. The exact solution obtained represents the generalization, for EYMH system, of the solutions previously obtained by Bais and Russell [6], by Cho and Freund [7]. In fact from a physical point of view our solution seems to be a more realistic candidates for the study of properties of collapsing objects.

\section{BASIC EQUATIONS AND SOLUTIONS}

We consider $S O(3)$ gauge theory with structure constant $C_{(\beta)(\gamma)}^{(\alpha)}$, the YM fields $F_{a b}^{(\alpha)}$, triplet of YM field $A_{a}^{(\alpha)}$ and a Higgs triplet $\phi^{(\alpha)}(a=0, \ldots, 3$ space-time indices and $\alpha=1,2,3$ isospace indices). The standard EinsteinHilbert action becomes 


$$
\mathcal{I}=\int \sqrt{-g} d^{4} x\left[-\frac{1}{2} g^{a b} g^{s d} F_{a s}^{(\alpha)} F_{b d}^{(\alpha)}-g^{a b}\left(D_{a} \phi^{(\alpha)}\right)\left(D_{b} \phi^{(\alpha)}\right)-\mu^{2}\left(\phi^{(\alpha)} \phi_{(\alpha)}\right)-\frac{\lambda}{4}\left(\phi^{(\alpha)} \phi_{(\alpha)}\right)^{2}\right]+\mathcal{I}_{N}
$$

Here, $g=\operatorname{det}\left(g_{a b}\right)$ is the determinant of the metric tensor and $I_{\mathcal{N}}$ is the action of null fluid. The classical equation of motion for YM fields are

$$
F_{a b}^{(\alpha)}=\partial_{a} A_{b}^{(\alpha)}-\partial_{b} A_{a}^{(\alpha)}+e \epsilon_{(\beta)(\gamma)}^{(\alpha)} A_{a}^{(\beta)} A_{b}^{(\gamma)} .
$$

The gauge covariant derivative $D_{a} \phi^{(\alpha)}$ is

$$
D_{a} \phi^{(\alpha)}=\partial_{a} \phi^{(\alpha)}+e \epsilon_{(\beta)(\gamma)}^{(\alpha)} A_{a}^{(\beta)} \phi^{(\gamma)} .
$$

where $\epsilon_{(\beta)(\gamma)}^{(\alpha)}$ is totally antisymmetric tensor. Variation of the action (1) with respect to the metric $g_{a b}$, gauge field $A_{a}^{(\alpha)}$ and Higgs field $\phi^{(\alpha)}$ leads to Einstein equation and matter field equations. Higgs field vacuum expectation value $|\langle\phi\rangle|=F$, where

$$
F^{2}=-\frac{2 \mu^{2}}{\lambda}
$$

The mass of the Higgs particle is given by $M_{H}=\sqrt{\lambda} F$. For the gauge and Higgs fields we employ the following ansatz:

$$
A_{a}^{(\alpha)}=\epsilon_{a(\beta)}^{b(\alpha)} \eta_{b} r^{(\beta)} A(r), \quad \phi^{(\alpha)}=r^{(\alpha)} \phi(r) .
$$

where $\eta_{b}=\delta_{b}^{v}$ is timelike unit vector. To construct spherically symmetric gravitating monopole solution in Vaidya space-time we employ the Eddington coordinates and adapt the metric of general spherically symmetric space-time [16 18] given by

$$
d s^{2}=-A(v, r)^{2} f(v, r) d v^{2}+2 \epsilon A(v, r) d v d r+r^{2} d \Omega^{2}
$$

, where $d \Omega^{2}=d \theta^{2}+\sin ^{2} \theta d \phi^{2}$. Here $A(v, r)$ is an arbitrary function. It is the field equation $G_{1}^{0}=0$ that leads to $A(v, r)=g(v)$. However, by introducing another null coordinate $\bar{v}=\int g(v) d v$, we can always set, without the loss of generality, $A(v, r)=1$. Therefore the entire family of solutions we are searching for is determined by a single function $f(v, r)$.
An exact solution for $A(r)$ and $\phi(r)$ are determined as

$$
\phi(r)=\frac{F}{r}, \quad \phi^{(\alpha)}=\frac{r^{(\alpha)}}{r} F
$$

and

$$
A(r)=-\frac{1}{e r^{2}}, \quad A_{a}^{(\alpha)}=\epsilon_{a(\beta)}^{b(\alpha)} \eta_{b} \frac{r^{(\beta)}}{r} \frac{1}{e r} .
$$

That such a solution corresponds to a magnetic monopole can be seen by inserting it into gauge-invariant generalization of the electromagnetic field tensor:

$$
\mathbf{F}_{a b}=\frac{\phi_{(\alpha)}}{|\phi|} F_{a b}^{(\alpha)}-\frac{1}{e} \epsilon_{(\alpha)(\beta)(\gamma)} \frac{\phi^{(\alpha)}}{|\phi|}\left(D_{a} \phi^{(\beta)}\right)\left(D_{b} \phi^{(\gamma)}\right)
$$

which yields

$$
\mathbf{F}_{a b}=-\epsilon_{a b(\alpha)} \frac{r^{(\alpha)}}{r^{3}}
$$

Clearly $\mathbf{F}_{a b}$ satisfies Maxwell equations, except at $r=0$, and corresponds to magnetic field

$$
\vec{B}=Q \frac{\vec{r}}{r^{3}}
$$

of a magnetic point charge with $Q=1 / e$. In this letter, without loss of generality, we choose $Q=Q(v)$. It is seen that, for the metric (6), the matter field equation admits solution $Q(v)=1 / e$. The Einstein equations is

$$
G_{a b}=T_{a b} .
$$

Expressing the total energy-momentum tensor (EMT) as

$$
T_{a b}=T_{a b}^{G}+T_{a b}^{N},
$$

where the gauge EMT $T_{a b}^{G}$ is

$$
\begin{aligned}
T_{a b}^{G}= & 2\left[g^{m n} F_{a m}^{(\alpha)} F_{b n}^{(\alpha)}-\frac{1}{4} g_{a b} g^{m n} g^{s t} F_{m s}^{(\alpha)} F_{n t}^{(\alpha)}+\left(D_{a} \phi^{(\alpha)}\right)\left(D_{b} \phi^{(\alpha)}\right)\right. \\
& \left.-\frac{1}{2} g_{a b} g^{m n}\left(D_{m} \phi^{(\alpha)}\right)\left(D_{n} \phi^{(\alpha)}\right)-g_{a b}\left(\frac{1}{2} \mu^{2}\left(\phi^{(\alpha)} \phi_{(\alpha)}\right)+\frac{\lambda}{8}\left(\phi^{(\alpha)} \phi_{(\alpha)}\right)^{2}\right)\right]
\end{aligned}
$$


and the null fluid EMT is

$$
T_{a b}^{N}=\psi(v, r) l_{a} l_{b}
$$

with $\psi(v, r)$, the nonzero energy density and $l_{a}$ is a null vector such that $l_{a}=\delta_{a}^{0}, l_{a} l^{a}=0$. For $a \neq b, T_{b}^{a}=0$ except for a nonzero off diagonal component $T_{v}^{r}$. It may be recalled that EMT of a Type II fluid has a double null eigenvector, whereas an EMT of a Type I fluid has only one timelike eigenvector [19]. In addition, we observe that the metric (6) requires that $T_{v}^{v}=T_{r}^{r}$.

Inserting Eqs. (77) and (8), we obtain the expression for the gauge-field tensor which in spherically coordinates becomes

$$
\begin{aligned}
F_{\theta \phi}^{(x)} & =-Q(v) \sin ^{2} \theta \cos \phi \\
F_{\theta \phi}^{(y)} & =Q(v) \sin ^{2} \theta \sin \phi \\
F_{\theta \phi}^{(z)} & =-Q(v) \sin \theta \sin \phi
\end{aligned}
$$

all other components vanish. Putting this in expression for EMT and setting $\beta=\mu^{4} / \lambda$, the EMT can be written as:

$$
T_{b}^{a}=\left(\begin{array}{cccc}
-\frac{Q^{2}(v)}{r^{4}}+\beta & 0 & 0 & 0 \\
\psi(v, r) & -\frac{Q^{2}(v)}{r^{4}}+\beta & 0 & 0 \\
0 & 0 & \frac{Q^{2}(v)}{r^{4}}+\beta & 0 \\
0 & 0 & 0 & \frac{Q^{2}(v)}{r^{4}}+\beta
\end{array}\right) .
$$

Here $\beta$ is contribution from scalar field due to spontaneous symmetry breaking. In the limit, $\beta=0$, EMT is same as in charged null fluid because $D_{a} \phi^{\alpha}$ vanish everywhere and does not contribute to EMT.

For the EMT (13) and with the metric (6), the Einstein equations (12) reduce to:

$$
\begin{array}{r}
\psi=-\frac{1}{r} \frac{\partial f(v, r)}{\partial v}, \\
\frac{1}{r} \frac{\partial f(v, r)}{\partial r}-\frac{1}{r^{2}}+\frac{f(v, r)}{r^{2}}=-\frac{Q^{2}(v)}{r^{4}}+\beta \\
\frac{1}{2} \frac{\partial^{2} f(v, r)}{\partial r^{2}}+\frac{1}{r} \frac{\partial f(v, r)}{\partial r}=\frac{Q^{2}(v)}{r^{4}}+\beta
\end{array}
$$

It may be noted that in view of Eq. (3), the gauge field has only the angular components nonzero and they go as $r^{-2}$ which in turn makes $T_{a b}^{G}$ go as $r^{-4}$. The null fluid part will be given by $T_{v}^{r}=\psi(r, v)$. The last two equations are not independent and it suffices to integrate Eq. (20b) to give

$$
f(v, r)=1-\frac{2 M(v)}{r}+\frac{Q^{2}(v)}{r^{2}}+\beta \frac{r^{2}}{3}
$$

where $M(v)$ is an arbitrary function of $v$. Since YM $T_{a b}^{G}$ go as $r^{-4}$ (the same as for Maxwell field in $N=4$ ), that is why its contribution in $f$ as in 4-dimensional ReissnerNordström static or Bonnor-Vaidya radiating black-hole
21]. Thus the metric describing the in $(v, r, \theta, \phi)$ coordinates reads as:

$$
\begin{array}{r}
d s^{2}=-\left(1-\frac{2 M(v)}{r}+\frac{Q^{2}(v)}{r^{2}}+\beta \frac{r^{2}}{3}\right) d v^{2} \\
+2 d v d r+r^{2} d \Omega^{2}
\end{array}
$$

From Eq. (20a), we obtain the energy density of the null dust with gauge charge as

$$
\psi(v, r)=\frac{2}{r^{2}} \frac{d M(v)}{d v}-\frac{2 Q(v)}{r^{3}} \frac{d Q(v)}{d v}
$$

and YM energy density and transverse stress are given by

$$
\begin{aligned}
\zeta(v, r) & =\frac{Q^{2}(v)}{r^{4}}-\beta \\
P(v, r) & =\frac{Q^{2}(v)}{r^{4}}+\beta
\end{aligned}
$$

The family of solutions discussed here, in general, belongs to Type II fluid defined in [19]. These are same results as one would expect for charge null dust in the Abelian theory, i.e., the geometry is precisely of the Bonnor-Vaidyade Sitter [15, 20] form and the charge that determines the geometry is YM gauge charge, whereas the Higgs field $\beta$ playing the role of a cosmological constant, from a formal mathematical point of view. Thus we can also say that any solution of the Einstein-Maxwell system is also solution of the Einstein-Yang-Mills (EYM) system.

The Kretschmann scalar $\left(K=R_{a b c d} R^{a b c d}, R_{a b c d}\right.$ is the Riemann tensor) for the metric (22) reduces to

$$
K=\frac{48}{r^{6}}\left[M^{2}(v)-\frac{2}{r} Q^{2}(v) M(v)+\frac{7}{6} \frac{Q^{4}(v)}{r^{2}}\right]+\frac{8}{3} \beta^{2}
$$

So the Kretschmann scalar diverges along $r=0$. The Weyl scalar $\left(C=C_{a b c d} C^{a b c d}, C_{a b c d}\right.$ is the Weyl tensor) reads

$$
C=\frac{48}{r^{6}}\left[M^{2}(v)-\frac{2 M(v) Q^{2}(v)}{r}+\frac{Q^{4}(v)}{r^{2}}\right]
$$

which also diverges along $r=0$.

In the rest frame associated with the observer, the energy-density of the matter will be given by,

$$
\psi=T_{v}^{r}, \quad \zeta=-T_{t}^{t}=-T_{r}^{r}=\frac{Q^{2}(v)}{r^{4}}-\beta,
$$

and the principal pressures are $P_{i}=T_{i}^{i}$ (no sum convention).

a) The weak energy conditions (WEC): The EMT obeys inequality $T_{a b} w^{a} w^{b} \geq 0$ for any timelike vector, i.e.,

$$
\psi \geq 0, \quad \zeta \geq 0, \quad P_{\theta} \geq 0, \quad P_{\phi} \geq 0 .
$$

We say that strong energy condition (SEC), holds for Type II fluid if, Eq. (29) is true., i.e., both WEC and 
SEC, for a Type II fluid, are identical.

b) The dominant energy conditions : For any timelike vector $w_{a}, T^{a b} w_{a} w_{b} \geq 0$, and $T^{a b} w_{a}$ is nonspacelike vector, i.e.,

$$
\psi \geq 0, \quad \zeta \geq P_{\theta}, P_{\phi} \geq 0 .
$$

Clearly, $(a)$ is satisfied if $Q^{2}(v) \geq \beta r^{4}$. However, $\psi>0$ gives the restriction on the choice of the functions $M(v)$ and $Q(v)$. From Eq. (28), we observe $\psi>0$ requires,

$$
\frac{2}{r^{2}} \frac{d M(v)}{d v}>\frac{2 Q(v)}{r^{3}} \frac{d Q(v)}{d v}
$$

We note that the stress tensor in general may not obey the weak energy condition. In particular, if $d M / d Q>0$ then there always exists a critical radius $r_{c}=Q \dot{Q} / \dot{M}$ such that when $r<r_{c}$ the weak energy condition is always violated. On the other hand, the DEC may not hold.

Thus, we have constructed an explicit nonstatic magnetically charged null fluid solutions of a non-Abelian gauge theory coupled to gravitation and Higgs field. Thus we have exact Vaidya like solutions of the EinsteinYang-Mills-Higgs model. This yields same results as one would expect for a charge null fluid in the Abelian theory, i.e., the geometry is precisely of the Bonnor-Vaidya form and the charge that determines the geometry is magnetic charge $(1 / e)$ with Higgs field contributing to cosmological constant like term. The geometry becomes asymptotically flat in the absence of the scalar field. Thus we found generalization of the model discussed previously by Bais and Russell [6] and independently by Cho and Freund [7]. Now a few comments are in order: (1) If one adds cosmological term to the Lagrangian, then the solution (21) requires additional term:

$$
f(v, r)=1-\frac{2 M(v)}{r}+\frac{Q^{2}(v)}{r^{2}}-(\Lambda-\beta) \frac{r^{2}}{3}
$$

It may be noted that Eq. (32) has three types of solutions, namely, Bonnor-Vaidya-de Sitter, Bonnor-Vaidya and Bonnor-Vaidya-anti-de Sitter solutions depending on whether $\Lambda>\beta, \Lambda=\beta$, or $\Lambda<\beta$ respectively. (2) Further, when the magnetic charge is switched off, i.e. if $Q^{2}(v)=0$, then, from Eq. (22), one obtains Vaidya-de Sitter metric and if both $Q^{2}(v)=\beta=0$ one gets Vaidya metric. (3) Another case is obtained if both $M=Q=$ constant. By introducing the transformation

$$
d t=d v-\left(1-\frac{2 M}{r}+\frac{Q^{2}}{r^{2}}+\beta \frac{r^{2}}{3}\right)^{-1} d r
$$

Eq. (22) becomes

$$
\begin{array}{r}
d s^{2}=-\left(1-\frac{2 M}{r}+\frac{Q^{2}}{r^{2}}+\beta \frac{r^{2}}{3}\right) d t^{2} \\
+\left(1-\frac{2 M}{r}+\frac{Q^{2}}{r^{2}}+\beta \frac{r^{2}}{3}\right)^{-1} d r^{2}+r^{2} d \Omega^{2}
\end{array}
$$

which is the same as that given by Bais and Russell [6] and Cho and Freund [7]. Thus, as mentioned earlier, several known models can be recovered from our analysis.

\section{CONCLUDING REMARKS}

Because of the complex nature of the full Einstein equations, the metrics with special symmetries are used to construct gravitational collapse models. One such case is the two-dimensional reduction of general relativity obtained by imposing spherical symmetry. Even with this reduction, however, very few inhomogeneous exact nonstatic solutions have been found. One well-known example is the Vaidya metric 14. In view of this, the solutions presented here can be useful to get insights into more general gravitational collapse situations and to model the dynamical evolution of a Hawking evaporating black holes. It would be useful to investigate the back reaction of an evaporating black hole with magneticmonopoles and also the changes in the structure and location of the horizons when a black hole with magnetic monopole radiates. Our solution can be also utilized to study Vaidya collapse with a magnetic monopole field and examine the formation of black holes and naked singularities and examine how the perturbation induced by the external matter fields affect the formation or otherwise of the naked singularity. The relevant question is whether the effect of such external fields could remove the occurrence of the same. Indeed, one can claim that the space-time discussed here has same singularity behavior as the Bonnor-Vaidya-de Sitter [20] due to mathematical similarity of our solution with that of Bonnor-Vaidya-de Sitter. For Bonnor-Vaidya-de Sitter case, shell focusing strong curvature naked singularities do arise and hence in our model as well the naked singularity definitely develops. In that sense, the gauge charge does not remove the naked singularity, which thus displays stability with respect to this particular mode of perturbation. The usefulness of these models is that they do offer opportunity to explore of properties of singular space-time and, in the case of curvature singularity to address issue such as local or global nakedness and strength. These and other related topics are under investigation [21].

It also serves to illustrate the much richer interplay that can occur among particle physics and general relativity when more involved theoretical models are considered. As a final remark, it would be also interesting to see how the results get modified in higher dimensional spacetime with the Gauss-Bonnet combination of quadratic curvature terms [21].

\section{Acknowledgments}

One of the authors SGG is supported by university grant commission (UGC) major research project grant F. NO. 39-459/2010 (SR). 
[1] P. A. M. Dirac, Proc. Roy. Soc. London A 133, 60, (1931).

[2] G. 't Hooft, Nucl. Phys. B 79, 276 (1974).

[3] A. M. Polyakov, JETP Lett. 20, 194 (1974).

[4] T. T. Wu and C. N. Yang, in Properties of Matter Under Unusual conditions, edited by H. Mark and S. Fernbach (Interscience, New York, 1969), p. 349.

[5] P. B. Yasskin, Phys. Rev. D 12 (1975) 2212.

[6] F. A. Bais and R. J. Russell, Phys. Rev. D 11, 2692 (1975).

[7] Y. M. Cho and P. G. O. Freund, Phys. Rev. D 12, 1588 (1975).

[8] P. van Nieuwenhuizen, D. Wilkinson and M. J. Perry, Phys. Rev. D 13, 778 (1976).

[9] M. E. Ortiz, Phys. Rev. D 45, R2586 (1992).

[10] N. A. Viet and K. C. Wali, Phys. Rev. D 51, 1664 (1995).

[11] S. Habib Mazharimousavi and M. Halilsoy, Phys. Rev. D 76, 087501 (2007).

[12] S. Habib Mazharimousavi and M. Halilsoy, JCAP 0812,
005 (2008).

[13] S. H. Mazharimousavi and M. Halilsoy, Phys. Lett. B 659, 471 (2008)

[14] P.C. Vaidya, Proc. Indian Acad. Sci. A33, 264 (1951) Reprinted, Gen. Rel. Grav. 31, 119 (1999).

[15] W. B. Bonnor and P. C. Vaidya, Gen. Rel. Grav. 1, 159 (1970).

[16] A. Wang and Y. Yu Gen. Rel. Grav. 31, 107, (1999).

[17] A. K. Dawood and S. G. Ghosh Phys. Rev. D 70, 104010 (2004).

[18] S. G. Ghosh and D. Kothawala, Gen. Rel. Grav. 40, 9 (2008).

[19] S. W. Hawking and G. F. R. Ellis The Large Scale Structure of Space-time (Cambridge University Press, Cambridge, 1973).

[20] K. Lake and T. Zannias, Phys. Rev. D43, 1798 (1991).

[21] S. G. Ghosh and N. Dadhich (work in progress). 